\title{
Problématiser et traiter la question de la chute du Mur trente ans après auprès d'un public étudiant en France : enjeux et méthodes
}

\author{
Elisa Goudin-Steinmann
}

Université Paris 3 Sorbonne Nouvelle

\section{Abstract}

Wie können wir dreißig Jahre nach dem Fall der Berliner Mauer einen angemessenen Blickwinkel finden, um ein möglichst genaues Bild der DDR in Frankreich zu vermitteln? Wie können wir diese Frage problematisieren, um sie auf originellere Weise anzugehen als nur durch das Studium des Repressionsapparates? Wie können wir uns von der Erzählung der 'Erfolgsgeschichte' der Bundesrepublik und der 'Misserfolgsgeschichte' der DDR, die ihr Gegenstück wäre, distanzieren? Wir schlagen mehrere Wege zur Eröffnung dieser Debatte vor, in Form von drei Leitgedanken: sich auf die Akteure konzentrieren, die Bedeutung des dritten Wegs betonen und die Ergebnisse der neueren Forschung über die DDR benutzen, da die Geschichte der DDR heute meist nicht mehr so geschrieben wird wie es in den 1990er Jahren der Fall war.

Trente ans après la chute du Mur, comment trouver un angle adéquat pour véhiculer en France les représentations les plus justes possibles sur ce qu'a été la RDA ? Comment problématiser cette question pour l'aborder de façon plus originale que la seule étude de l'appareil de répression ? Comment sortir du récit de la Erfolgsgeschichte de la République fédérale et de la Misserfolgsgeschichte de la RDA qui en serait le pendant? Nous proposons plusieurs pistes pour ouvrir ce débat, sous la forme de trois idées directrices : mettre l'accent sur les acteurs, sur la notion de troisième voie, et utiliser les résultats de la recherche récente sur la RDA, car on n'écrit pas aujourd'hui l'histoire de la RDA comme dans les années 1990.

Thirty years after the fall of the Wall, how can we find an adequate angle to convey the best representations of what the GDR was like in France? How can we problematize this question in order to approach it in a more original way than just studying the Stasi? How can we get away from the narrative of the Erfolgsgeschichte of the Federal Republic and the Misserfolgsgeschichte of the GDR, which would be its counterpart? We propose several possibilities in order to open this debate, in the form of three guiding ideas, focusing on the actors, emphasizing the notion of a third way, and using the results of recent research on the GDR, because the history of the GDR is no longer written today as it was in the 1990s.

\section{Keywords}

DDR im Unterricht • Fall der Berliner Mauer • dritter Weg • Forschung über die DDR-Geschichte

Enseignement sur la RDA • chute du Mur de Berlin • troisième voie • recherche sur la RDA

Teaching the GDR • fall of the Wall in Berlin • third way • research on the GDR

Une étude publiée en 2001 avait causé beaucoup d'inquiétude concernant le niveau de connaissances des étudiant.e.s allemands sur l'histoire de la RDA (Pasternack 2001), comme le montre par exemple l'article qu'avait publié la taz le 23 janvier 2002 : « Erich Honecker? Nie gehört! » (Erich Honecker? Jamais entendu parler !). A cela s'est ajouté le débat autour de l'idée que la RDA n'aurait été qu'une « note de bas de page de l'histoire " (Faulenbach 2003), pour reprendre le terme utilisé par l'historien Hans-Ulrich Wehler (qui reprenait une expression de Stefan Heym). Autour du tournant des années 2000 , on s'est beaucoup préoccupé de la question de l'ignorance de l'histoire de cet État, réduit à néant une seconde fois par sa disparition comme objet d'enseignement ${ }^{1}$. Trente ans après la chute du Mur, cette thématique est moins présente dans les médias allemands mais la question de savoir comment trouver un angle adéquat pour véhiculer les représentations les plus justes possibles sur ce qu'a été la

1 II existe par exemple un site internet dédié à l'enseignement de la RDA en Allemagne dans les cours d'histoire, https://www.ddr-im-unterricht.de, mis en place à l'initiative de la Landeszentrale für politische Bildung Baden-Württemberg, donc un Land de l'Ouest. II y est surtout question du fonctionnement de l'Etat, de l'appareil de répression, de l'idéologie, et un peu également de la vie quotidienne et du sport par exemple. 
RDA, demeure posée. Elle se pose de façon un peu différente dans les nouveaux Länder, où le récit familial vient souvent compléter ou infléchir l'enseignement dispensé à l'Université. Mais partout il s'agit d'un enjeu important pour l'avenir. En France elle demeure, du fait de l'absence de proximité avec l'objet d'études, une question ouverte à laquelle nous voudrions apporter quelques pistes de réponses ici.

En effet, d'ici quelques années, plus de temps se sera écoulé depuis la chute du Mur que la durée d'existence de la RDA. Dès lors comment faire de cet État, qui est bien souvent pour les lycéen.ne.s et les étudiant.e.s un simple objet historique, un thème d'enseignement (Pilon 2012) ? Comment traiter la question de la chute du Mur trente ans après, dans un pays comme la France ${ }^{2}$ ? Comment problématiser cette question pour l'aborder de façon plus originale que la seule étude du système politique, du système économique, en un mot de la seule étude de l'appareil de répression ? Comment sortir du récit de la Erfolgsgeschichte de la République fédérale et de la Misserfolgsgeschichte de la DDR qui en serait le pendant?

\section{IDÉE 1 : METTRE L'ACCENT SUR LES ACTEURS}

Dans son doctorat soutenu récemment, Marie MüllerZetzsche (2018) compare la façon dont on enseigne l'histoire de la RDA en Allemagne de l'Ouest, en Allemagne de l'Est et en France ${ }^{3}$. Grâce à cette comparaison, les mécanismes de mémoire collective et d'appropriation des objets d'enseignement deviennent visibles. Or, elle montre qu'il n'existe pas de relation causale entre le manque de savoir et la banalisation de la RDA. Selon les résultats de son enquête, « l'interprétation d'une RDA minimisée, défendue par les élèves à Leipzig, est basée sur la culture matérielle de l'État disparu et n'exclut pas l'interprétation de la RDA comme «État SED ». Dans la classe est-allemande, la banalisation de la RDA par les élèves n'apparaît pas comme étant le résultat de leur ignorance mais plutôt comme étant, d'une part, de l'attachement à l'héritage dévalorisé de la RDA, et, d'autre part, le reflet de la loyauté exprimée par leurs parents et grands-parents » (ibid).

C'est la première piste que nous proposons pour transmettre des connaissances plus fines sur la RDA : mettre l'accent sur les acteurs, pas seulement sur ceux qui ont façonné la vie politique ou économique, mais aussi sur les simples citoyens, montrer des trajectoires de vie, en incluant la façon dont les personnes se sont adaptées au changement après 1989 avec plus ou moins de difficultés. Pour apporter à l'enseignement sur la RDA cette densité biographique, on peut s'appuyer sur l'ouvrage d'Agnès Arp et d'Annette Leo, qui retranscrit

2 II n'existe pas à notre connaissance d'étude globale sur la place de la RDA dan l'enseignement secondaire ou universitaire en France, qui analyserait en détail la place de cet objet d'enseignement dans les maquettes des différentes universités par exemple. Mais nous renvoyons à l'ouvrage déjà un peu ancien de Catherine Fabre-Renault, Élisa Goudin, Carola Hähnel-Mesnard (2006) qui établissait un premier état des lieux. Voir aussi Appenzeller (2008).

3 Thèse soutenue en 2018 à Leipzig sous la direction de Ulrich Pfeil et Thomas Höpel.
16 récits de vie de citoyens de RDA venus d'horizons divers (Arp / Leo 2009) 4 .

Une telle approche permet de montrer comment la logique de cet État perdait de plus en plus son sens pour une partie de la population, mais que pourtant personne n'aurait parié sur son écroulement aussi brutal et surtout aussi rapide. Tout s'est désagrégé très vite et sans grande explosion, sans intervention des chars soviétiques, grâce notamment à Mickaïl Gorbatchev qui s'y est opposé, contre l'avis de certains membres de son gouvernement. Les récits de vie ne sont pas seulement une source d'histoire orale pour les chercheurs, ils peuvent aussi être un matériau pédagogique précieux pour faire comprendre ce qu'était la vie quotidienne en RDA et l'expérience de l'effondrement de cet État.

Cela permet également d'expliquer aux étudiant.e.s que le chancelier ouest-allemand Helmut Kohl, qui pouvait se prévaloir d'une légitimité en raison de la victoire du Parti $A /$ lianz für Deutschland aux élections du 18 mars 1990, a fait le choix de ne pas fonder un nouvel État, mais d'utiliser, pour aller plus vite, un article de la Loi fondamentale de RFA permettant de recueillir un nouveau Land au sein de la RFA (cet article avait été prévu pour la Sarre en 1945). De cette façon, la RFA n'a jamais cessé d'exister, elle a seulement « récupéré » cinq nouveaux Länder. Mais il est important de faire prendre conscience aux étudiant.e.s qu'une occasion de mener une réflexion commune sur les fondements d'un nouvel État a indéniablement été perdue à ce moment-là : si des juristes est-allemands et ouest-allemands avaient dû rédiger ensemble une nouvelle Constitution, ils auraient dû se confronter à certaines différences entre les deux systèmes, et modifier le droit de la nationalité (droit du sang comme en RFA ou droit du sol comme en RDA ?), la législation sur l'avortement (plus libérale à l'Est), la question du droit au logement et au travail qui étaient des garanties de droit en RDA pour les citoyens - autant de questions qui auraient éventuellement permis de prendre en compte certains aspects positifs de la politique sociale de la RDA ${ }^{5}$.

Au total, les choses sont allées si vite que le sentiment qu'une digue s'était rompue est partagé par de nombreux anciens citoyens de RDA, et cela se comprend très bien à la lecture des récits de vie ${ }^{6}$. II s'agit aussi de rendre possible, par l'étude de ces récits de vie, une mise en perspective par les étudiant.e.s de la distance de trente ans qui nous sépare de la fin de la RDA. On retrouve en effet très souvent dans les médias un discours qui voudrait faire croire que la RDA n'aurait finalement été qu'une sorte de bulle idéologique qui aurait éclaté, ne laissant aucune

4 Nous renvoyons également à l'ouvrage que nous publierons en 2020 chez Nouveau Monde éditions, avec Agnès Arp, autour de la RDA après la RDA, qui sera également basé sur des récits de vie.

5 La seule réforme du régime communiste qui resta exempte de pourparlers est la réforme agraire (Bodenreform) qui avait été conduite dans la zone d'occupation soviétique

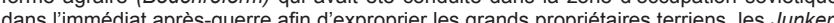
et de collectivise leurs teres. M. Gopatchev avait en effet posé cette condition sine qua non en échange de sa signature du traité d'unification.

6 Cela s'explique par la nette majorité obtenue par Allianz für Deutschland en mars 1990 qui a donné toute latitude au chancelier Kohl pour agir. 
trace, comme un accident de l'histoire corrigé il y a trente ans. Or, la mise en récit de leurs expériences par les principaux concernés, les anciens citoyens de RDA, permet de saisir l'écart entre ce type de discours et l'attachement à ce qui n'était pas nécessairement vécu comme un accident de l'histoire, mais parfois plutôt comme une Heimat, surtout rétrospectivement après 1989.

Dans le même ordre d'idées, on considère souvent que la RDA n'avait aucune cohérence territoriale ni aucune logique dans ses frontières. Dans les livres d'histoire, elle est toujours présentée comme un pays "croupion ", correspondant à la zone qui avait été attribuée à l'Union soviétique au moment du partage du gâteau allemand entre les Alliés ${ }^{7}$. En conséquence, les frontières de cet État seraient totalement arbitraires, résultant uniquement des positions militaires en 1945. Ce n'est pas vrai. II peut être utile d'expliquer aux étudiant.e.s que le territoire de RDA renvoie aussi à une réalité historique bien plus ancienne que l'histoire de la RDA. Son unité existe bel et bien, elle est ancrée dans une économie agraire qui avait des caractéristiques dues à une histoire particulière différente du reste des territoires germaniques. A peu de choses près, les frontières de la RDA correspondaient à celle du Mitteldeutschland, cette étendue entre l'Elbe et l'Oder, fermée au sud-ouest par le massif du Harz, au sud par la forêt de Thuringe et au sud-est par le Erzgebirge, les monts métallifères ${ }^{8}$. La spécificité de cette région du Mitteldeutschland est que presque partout depuis le XIlème siècle la Grundherrschaft a perduré : il s'agit d'un pouvoir fondé sur l'occupation foncière et sur une base contractuelle, et non pas un pouvoir personnel comme dans le reste des terres agricoles du territoire germanique. En ce sens, les frontières de la RDA renvoient à une logique historique précise sur le long terme, très antérieure à la RDA. Les logiques qui ont prévalu en 1945 pour la répartition des zones entre les Soviétiques et les Américains sont de ce fait à distinguer très clairement de la question des identités territoriales, imposant de remonter bien en amont de la guerre.

Enfin, centrer l'enseignement sur les acteurs plutôt que sur les institutions permet de comprendre pour quelles raisons, trente ans après, la RDA redevient une source d'inspiration pour les partisans d'une remise en cause du modèle néolibéral. Dans La fille qui venait d'un pays disparu, Saskia Hellmund écrit :

A l'heure d'une crise profonde du système du marché libre, les expériences et les savoir-faire des gens qui ont connu plus d'un modèle de société pourraient pourtant redevenir importants. Si on veut bien les écouter, ils vous parleront de la primauté du lien social. Ils vous raconteront comment

\footnotetext{
7 II sera intéressant de consulter les nouveaux manuels qui vont être utilisés pour l'enseignement de l'histoire au lycée suite à la réforme du bac, mais pour l'instant, même le manuel d'histoire franco-allemand adopte cette perspective. Voir aussi au sujet d'internet les travaux de Hein (2004).

8 Ces terres, à l'Est du Altdeutschland étaient habitées par des populations slaves et ont été occupées durant la première vague de colonisation organisée par l'Ordre teutonique à
} partir de 1105. on peut vivre heureux sans consommer. Ils vous diront la nécessité d'un État qui contrôle un minimum l'économie et qui planifie et investit suivant les besoins de la population. (Hellmund $2015: 57$ )

II faudrait évidemment discuter du fait que l'État investissait selon les « besoins de la population », ce qui était loin d'être toujours vrai. Mais on voit que la RDA est associée, dans son souvenir, avec la primauté du lien social, le dos tourné à la société de consommation (pourtant souvent rêvée et fantasmée à l'Est en raison de la possibilité de capter la télévision ouest-allemande et de se faire par ce biais une image totalement erronée de cette " société d'abondance »). Si les Allemands de l'Est sont " ostalgiques " c'est peutêtre avant tout de cette distance face à la recherche de croissance qui guide tous les choix sociaux et sociétaux. Ils ne regrettent en général pas la RDA elle-même, mais certaines pratiques sociales, et notamment cette distance face à la société de consommation, ce que l'on appellerait aujourd'hui la décroissance et qui va de pair avec le partage des richesses.

Or, tout ceci fait écho avec des thématiques extrêmement actuelles sur la nécessité de freiner le développement économique pour sauver la Planète de la catastrophe (la thématique du respect de l'environnement naturel était au cœur des milieux d'opposition en RDA), sur la nécessité de sortir de la course au toujours plus, toujours plus vite, sur l'idée que la recherche de la croissance, fût-elle " verte » serait devenue une idéologie à laquelle on sacrifie bien trop de choses.

\section{IDÉE 2 : METTRE L'ACCENT SUR LA NOTION DE TROISIĖME VOIE}

La formule de "l'effondrement de la RDA » (« Zusammenbruch der DDR ») couramment utilisée ne rend pas justice à la part active qu'ont pris les citoyens est-allemands à la révolution. Elle est problématique car elle ne prend pas en compte la lutte citoyenne à l'origine des transformations qui ont mené à la chute du Mur, le courage collectif qui a été nécessaire pour se rebeller (alors qu'en juin 1989, donc très peu de temps avant, les chars avaient été utilisés pour réprimer dans le sang la révolte de la place Tiananmen à Pékin). La RDA, même s'il est indéniable qu'elle était à bout de souffle, ne s'est pas simplement écroulée d'elle-même comme un château de cartes inanimé.

La période de l'automne 1989 a été très riche de ce point de vue : on fondait partout des tables rondes, des cercles citoyens, on signait des manifestes, on écrivait des programmes : on tentait d'inventer un monde nouveau en réfléchissant à l'avenir de la RDA. Toutes ces expériences peuvent se définir par le concept de " troisième voie ». Mais rien n'est resté de ces propositions de réformes en raison des élections de mars 1990, déjà mentionnées, qui ont donné toute liberté d'action à Helmut Kohl. Le fait que l'on ait demandé aux Allemands de l'Est de s'intégrer discrètement, sans rien pouvoir mettre dans la corbeille de mariage ou 
presque, a généré des frustrations qui existent aujourd'hui encore, et la conviction qu'il aurait été possible de faire autrement. Le sentiment de frustration est donc venu après, avec la violence de la désindustrialisation et de la délégitimation (Heukenkamp 2004).

Le sentiment d'appartenance commune, le " nous " des Allemands de l'Est tel qu'il se formule aujourd'hui, est né de cette expérience partagée d'une déception immense due à l'abandon de la politique sociale de RDA et à une crise économique d'une ampleur considérable, nettement pire à bien des égards que dans les autres démocraties populaires, à l'exception de la Bosnie-Herzégovine. Un seul chiffre permet d'en mesurer la profondeur : en 1994, le taux de natalité dans l'ex-RDA a atteint le chiffre de 0,77 enfant par femme ${ }^{9}$ (contre 1,35 pour les anciens Länder). Dans aucun autre État dans le monde (hormis le... Vatican !), à aucune époque on n'a enregistré un taux aussi bas. Le fait de ne plus se projeter dans la parentalité vient de l'incertitude sur l'avenir, de la précarité trop grande de la situation que vivaient de nombreux Allemands de l'Est ${ }^{10}$. Alors qu'en RFA la démocratie s'est installée avec le miracle économique, le plein emploi, la reconstruction et l'État-providence, à l'Est elle s'est installée avec le chômage massif et la délégitimation brutale de tout une période de vie. Cela fait une très grande différence. L'acquisition de droits citoyens et politiques est allée de pair avec une nouvelle insécurité sociale, économique, parfois existentielle, au lieu de se traduire par une meilleure protection contre les risques de l'existence, ce qui explique en partie les résultats électoraux toujours divergents entre l'Est et l'Ouest. Dans certaines villes du nord de l'ex-RDA, le chômage a touché 30 à $40 \%$ des habitants en âge de travailler - une situation totalement nouvelle pour ces personnes, et particulièrement douloureuse, dans la mesure où la RDA était une société très fortement marquée par l'idéal du travail. II est important d'expliquer aux étudiant.e.s que le travail structurait une grande partie de la vie sociale en RDA, à travers la brigade ${ }^{11}$, le collectif de bureau, les sorties communes après le travail pour se rendre à des spectacles, les ateliers artistiques dans les entreprises, tous ces éléments qui créaient du lien social. C'est aussi dans le milieu du travail que pour une part la vie amicale et les loisirs s'organisaient, à travers les crèches d'entreprise, les clubs sportifs amateurs, les distractions diverses, excursions, fêtes d'anniversaire.. En 1997, la Volksbühne de Berlin a fait salle comble avec un spectacle au titre évocateur: "La liberté rend pauvre » (Freiheit macht arm). Son metteur en scène, Frank Castorf, présentait la RFA comme un État qui condamne

9 Source : Zusammengefasste Geburtenziffer: Entwicklung der Fertilitätsrate in Deutschland von 1990 bis 2018, https://de.statista.com/statistik/daten/studie/36672/umfrage/anzahl-der-kinder-je-frau-in-deutschland/

10 Cette statistique ne prend évidemment pas en compte le fait que certains Allemand de l'Est sont partis à l'Ouest et y ont fondé des familles, ce qui est aussi une conséquence indirecte de la crise économique subie à l'Est.

11 Les travaux de Sandrine Kott $(2001 ; 2000 ; 1999)$ montrent bien dans quelle mesure la brigade était aussi un instrument de contrôle social. systématiquement tout ce qui ressemble de près ou de loin au collectif, et la pièce opposait l'individualisme qui appauvrit au collectif qui rend riche. De plus, avec les mutations sur le marché du travail, c'est toute une échelle de valeurs qui a été remise en cause brutalement ${ }^{12}$, ce qui a créé parfois des problèmes psychologiques importants : "Héros du travail hier, le mineur et l'ouvrier sidérurgiste sont devenus du jour au lendemain des "pollueurs », responsables de la destruction de l'environnement ${ }^{13}$ ». Faire prendre conscience aux étudiant.e.s de ces enjeux propres à l'histoire de la RDA permet de sortir de la seule étude de cet État comme une dictature orchestrée par le Parti et la Stasi.

Il est possible, dans le prolongement de ces explications, de poser la question des possibles non advenus, de la façon dont l'unification aurait pu ou non être conduite autrement. Un des exemples est bien sûr la Treuhand, chargée d'environ 8000 entreprises à restructurer ou privatiser ${ }^{14}$. Au total, même si cette dernière a permis l'émergence de quelques entreprises est-allemandes solides et performantes, le transfert de richesses de l'Est vers l'Ouest a été considérable : $80 \%$ des biens industriels administrés par la Treuhand sont aujourd'hui détenus par des Allemands de l'Ouest (au sens d'Allemands vivant actuellement à l'Ouest). Par ailleurs, grâce au système de dégrèvement d'impôt de $50 \%$ de la valeur des biens, près des trois quarts du foncier et des immeubles sur le territoire de l'ex-RDA appartiennent aujourd'hui à des Allemands de l'Ouest. Là encore, d'autres arbitrages auraient sans doute été possibles. En cédant les entreprises de l'ex-RDA non pas aux plus offrants mais - du moins officiellement - à ceux qui proposaient les meilleures garanties pour la poursuite de l'activité, la Treuhand s'est basée sur des critères de choix subjectifs qui ont rendu ses décisions opaques sans pour autant tenir la promesse de sauvegarde de l'emploi.

Une autre asymétrie évidente concerne l'indemnisation des anciens prisonniers. Selon l'article 131 du traité d'unification, l'indemnisation pour déportation pendant le national-socialisme ne s'applique pas aux nouveaux Länder, donc les citoyens de l'ex-RDA n'y ont pas droit. On pourrait multiplier à l'envi les exemples de gestion asymétrique mobilisables auprès des étudiant.e.s.

Ces arbitrages se sont accompagnés de clichés véhiculés sur la société est-allemande. En 2000, un criminologue et professeur de droit de Hanovre, Christian Pfeiffer, a été jusqu'à faire le lien entre la surreprésentation des Skinheads à l'Est et le fait que l'on avait mis les enfants collectivement sur le pot dans les jardins d'enfants de RDA (Siemens 2015) ! Selon lui, il s'agissait d'une logique collective qui

12 Cela fait aussi écho à des périodes de rupture semblables dans d'autres territoires avec la fermeture des mines de charbon en Grande-Bretagne ou en France, mais aussi dans la Ruhr par exemple.

13 Voir à ce sujet la réflexion de Jean Mortier à partir de l'ouvrage de Petra Köpping (2018), https://allemagnest.hypotheses.org/1478 [consulté le 14/09/2019].

14 Nous renvoyons à la très belle exposition itinérante de la Rosa-Luxemburg-Stiftung qui part de parcours biographiques : https://www.rosalux.de/dossiers/1989-aufbruch-insungewisse/schicksal-treuhand-schicksale/ [consulté le 14/09/2019]. 
expliquait la dérive vers la violence des enfants concernés, la militarisation du régime en RDA n'ayant pas permis de tenir compte des individualités. Cela lui a valu le surnom de Töpfchen-Pfeiffer ("Pfeiffer le pot»). Cette anecdote est parlante pour les étudiant.e.s : elle montre combien les clichés véhiculés contre l'Est et la volonté de se démarquer pouvaient aller loin. Pourtant, si l'on suit cette logique, les Allemands de l'Est auraient dû être plus favorables que les Allemands de l'Ouest à la guerre en Yougoslavie en 2000, la première guerre menée avec l'armée allemande depuis la fin de la Seconde Guerre mondiale. Ce fut tout le contraire, les sondages montrant qu'ils rejetaient massivement cette intervention militaire, davantage que leurs concitoyens de l'Ouest (Sarovic 2016).

Au total, l'idée est de faire réfléchir les étudiant.e.s - sans tomber dans un discours caricatural (« on aurait dû », « il fallait tout simplement »...) - aux enjeux de délégitimation de l'expérience de la RDA et des propositions de troisième voie ayant émergé lors de la Wende. En 1990, les Allemands de l'Est ont produit trois fois plus de déchets que leurs homologues de l'Ouest. On voulait tout jeter, se débarrasser de toute la RDA, tel un objet encombrant. C'est aussi ce qui explique le succès de Berlin dans les années 1990 comme ville alternative pour de nombreux artistes du monde enti$\mathrm{er}^{15}$, une ville où il était possible de se loger gratuitement en investissant un squat, et de se meubler en piochant tout simplement dans la rue, tant il y avait d'objets jetés ${ }^{16}$. Pourtant, le fait que la RDA ait été une dictature ne signifiait pas qu'aucun élément de son modèle de société ne méritait que l'on s'y attarde.

\section{IDÉE 3 : UTILISER LES RÉSULTATS DE LA RECHERCHE RÉCENTE SUR LA RDA DANS L'ENSEIGNEMENT}

On n'écrit plus du tout aujourd'hui l'histoire de la RDA comme dans les années 1990. Les historiens, sociologues, politologues, utilisent davantage les sources « d'en bas » (les syndicats, les organisations de masse, le sport amateur, les cercles artistiques dans les entreprises...) plutôt que les sources « d'en haut » comme les archives de la Stasi depuis le début des années 2000. Les connaissances sur la RDA ne se limitent plus au système économique de planification, aux modes de répression de la Stasi, ou à une comparaison de la dictature socialiste avec la dictature national-socialiste.

L'historien Jochen Staadt raconte dans un court article publié en 1996 l'énergie et le temps dépensés par le bureau politique du SED dans les années 1970 pour décider du bon dosage de chicorée dans le café vendu en RDA (Staadt 1996),

15 Ce succès de Berlin tenait aussi au fait que, justement, il restait des traces de la RDA, à la fois dans l'espace urbain, dans les manifestations culturelles proposées et grâce à la présence de très nombreux témoins de la RDA.

16 Cela vient aussi du fait que certains Allemands de l'Est qui avaient un peu d'argent de côté ont pu dépenser leurs économies tout à coup réévaluées en 1990 grâce au taux de change de 1 contre 1, pour acheter de nouveaux meubles, de nouveaux appareils électroménagers, etc. en raison du casse-tête que représentait la nécessité de réconcilier le goût des ménagères et la situation alarmante de la dette extérieure de la RDA à cette période. II montre ainsi à quel point le Parti était omniprésent et prenait en charge les problèmes du quotidien. On ne peut toutefois pas comprendre les mobilisations populaires de l'automne 1989 et la paralysie des centres de pouvoir à ce moment décisif si l'on s'arrête à ce constat d'une omniprésence du Parti dans la régulation des questions économiques, politiques, sociales et matérielles. II est nécessaire de comprendre les interactions multiples avec la société ${ }^{17}$

Au total, ces recherches "par en bas " sur le rôle joué par la société civile en RDA tendent à montrer que la politique du SED ressemblait davantage à une perpétuelle gestion de crise qu'à l'application d'un programme clair, univoque, prédéfini. Et il est utile de mobiliser quelques exemples concrets auprès des étudiant.e.s afin de leur transmettre une représentation de la société de RDA plus proche de la réalité que celle qui est véhiculée par les livres d'histoire : il existait évidemment certaines sphères du pouvoir dans lesquelles la rhétorique officielle ne souffrait aucune contradiction, mais il existait aussi des espaces où la parole était plus libre et où la réception des directives du Parti plus flexible. On connaît maintenant de nombreux cas de négociations de membres du bureau politique qui ont accepté de faire des compromis. L'exemple du rock est à cet égard très parlant : au début des années 1950, le SED a tout fait pour discréditer cette musique, l'interdire et harceler les fans de Elvis, puis ceux des Rolling Stones, des Beatles, avant de revoir sa position au cours des années 1960 en constatant que l'intérêt de la jeunesse ne faiblissait pas (Rauhut 1993). II a alors tenté de favoriser la naissance d'un rock est-allemand, avec un succès très relatif, mais il n'en demeure pas moins que la position de principe antirock en vigueur dans les années 1950 en RDA a été totalement abandonnée.

Dans le même ordre d'idées, les recherches de Peter Hübner (1994; 1995) sur les ouvriers dans la région industrielle de la Niederlausitz ont mis en lumière l'incapacité du SED et du syndicat FDGB à imposer les augmentations de cadences, les réductions de primes ou de salaires et l'introduction d'une rémunération à la tâche, en raison de l'opposition constante des ouvriers. De même, les travaux de Ralf Jessen sur le milieu universitaire ont mis en évidence les marges de manœuvre dont ont pu disposer certains chercheurs face au SED. Dans l'ensemble, tous ces travaux tentent, à partir d'un point de vue micro-historique, d'identifier aussi bien le processus de pénétration du Parti et de l'État dans la société, que les limites de leur emprise sur les citoyens.

Fanny Le Bonhomme, quant à elle, a travaillé sur la clinique psychiatrique de la Charité Berlin-Est. La psychiatrie est par essence un domaine où la parole est moins contrainte

17 Cela implique une périodisation fine de l'histoire de la RDA et une approche comparative avec d'autres pays, aussi bien du bloc de l'Est que de celui de l'Ouest. Nous renvoyons aux ouvrages suivants qui peuvent servir de référence à cet égard: Bessel / Jessen (1996) ; Dreyfus (2000) ; Droit / Kott (2006) ; Engler (2002) ; Lüdtke / Kalinowski (1998) Schmiechen-Ackermann (2002) : Silbermann / Wrage (2014). 
que dans le reste de la société. L'auteure a donc pu, à partir des dossiers de patients, saisir certaines des tensions qui traversaient la société est-allemande, les conflits intérieurs de personnes convaincues par le marxisme mais critiques envers le SED. Cette approche micro-historique est précieuse pour saisir le décalage entre le discours du SED et la réalité de sa réception par le public. On pourrait multiplier les exemples, tous ces résultats de recherche peuvent servir d'illustration dans le cadre d'un enseignement, pour montrer à la fois le fonctionnement du système et ses limites. L'idée plus générale, que les étudiant.e.s ont rarement en tête, surtout au début de leurs études, est que I'on a souvent tendance à subsumer les expériences des individus sous de grands énoncés comme "la guerre ", " l'industrialisation », " la dictature du SED », alors que ces grands schémas ne sont pas à même de rendre compte des représentations que cela véhiculait ni de ce que les individus vivaient réellement au quotidien (un paysan ouestallemand dans les années 1960 n'aurait de toute évidence pas été d'accord pour dire qu'il vivait dans la période dite du « miracle économique »...).

Afin de comprendre le concept de « dictature du SED » dans toutes ses nuances, il faut donc non seulement expliquer comment était structuré l'État en RDA, mais quels impacts cela avait sur la vie quotidienne, et les résultats de la recherche récente sont un outil pour cela. La démarche des historiens du quotidien, les Altagshistoriker a en effet transformé en profondeur la recherche sur la RDA. On sait désormais que la dictature de l'État-parti n'a pas été totale ${ }^{18}$ car elle n'est jamais parvenue à façonner une société indifférenciée, monolithique, une société qui aurait été « entièrement dominée de part en part " par le pouvoir politique, pour reprendre l'expression de Jürgen Kocka (Durchherrschung), ce qui contredit en partie la conviction que la société de RDA aurait été une société asphyxiée par le pouvoir, une société paralysée (stillgelegt) ${ }^{19}$ (Kocka 1994). Trente ans après, c'est l'un des enjeux cruciaux de l'enseignement de l'histoire de la RDA auprès des étudiant.e.s.

Dans son ouvrage sur les controverses publiques et privées autour des interprétations du passé de la RDA, Pamela Heß montre à l'aide d'une étude statistique portant sur la presse locale et régionale ainsi que sur des entretiens biographiques qu'il n'y a pas de consensus sur une mémoire commune, partagée, de la RDA (Heß 2014 : 195). Les mots-clés les plus récurrents relèvent du champ lexical de la domination de l'État : emprisonnement, dictature, soumission, Mur, fuite, victime. Puis viennent, mais loin derrière, les références à l'opposition, " opposition antifasciste », " révolution pacifique ». Le quotidien et tout ce qui a trait à la vie des citoyens n'intervient que plus rarement encore : la notion de « crèche, éducation des enfants " n'occupe que la 22ème place dans

18 Le concept de Eigensinn par exemple permet de questionner les limites de la capacité du SED à asphyxier la société (Lüdtke 1989). Pour une explication très complète de la genèse du concept et de son application en histoire, voir les travaux de Thomas Lindenberger (1999).

19 Voir sur cette tendance les travaux de la politiste Sigrid Meuschel (1993). les différentes catégories établies par Pamela Heß selon la fréquence des occurrences de ces termes, et la notion de " RDA sociale » occupe même la place 24 , soit la dernière dans la typologie de la chercheuse.

Pendant de longues années et encore en grande partie aujourd'hui en RFA, la recherche sur la RDA s'est organisée uniquement autour de sa mission pédagogique envers le grand public, avec l'idée sous-jacente que la connaissance du fonctionnement de la dictature permettrait une meilleure identification avec la démocratie et une volonté accrue de s'investir pour cette démocratie. Or, il est évident que ce lien n'est pas mécanique, et, même s'il l'était, il faudrait reconnaître que cela a totalement échoué : le racisme, le nationalisme, le sexisme, l'homophobie, sont aujourd'hui très présents notamment dans les nouveaux Länder, malgré cet effort pédagogique. Les succès récents de l'AfD dans ces régions ne font malheureusement que confirmer cela. II est capital de faire prendre conscience aux étudiant.e.s de ces enjeux : servir un objectif politique de légitimation de la République de Berlin par une lecture trop manichéenne des réalités sociales caractéristiques de l'ex-RDA est absurde.

\section{CONCLUSION}

II ne s'agit évidemment en aucun cas de minimiser le caractère dictatorial du régime du SED. Mais l'un des enjeux de l'enseignement de cette période de l'histoire allemande, trente ans après sa fin, est aussi de faire comprendre que si on part de l'idée que la société civile n'existait pas, qu'elle était totalement asphyxiée par le régime, alors on ne peut pas comprendre comment cette société a tout à coup été capable d'une telle mobilisation collective, capable de faire chuter le Mur, de mettre en avant autant de propositions de réformes (Jarausch 1995). Cela implique de recentrer le contenu de l'enseignement sur les acteurs de la vie quotidienne parallèlement à l'étude du fonctionnement de l'hégémonie du Parti, du système économique, des simulacres d'élections, des figures d'Ulbricht et d'Honecker. Cela permet aussi de ne pas homogénéiser la société de RDA, de montrer que tous n'ont pas vécu de la même manière en RDA, que tous n'ont pas été favorables à la chute du régime, n'ont pas fait les mêmes revendications politiques, l'étude des récits de vie montre qu'il y avait une pluralité des expériences irréductible à la seule prise en compte du milieu social par exemple. Plus largement, ce choix de se concentrer sur les acteurs de la vie quotidienne, sur les témoignages de citoyens " ordinaires " s'oppose à la conception qu'il existerait des personnes, des actes plus historiques que d'autres, une conception héritée d'une certaine approche du positivisme. Et cela doit aussi viser à contrer les discours de délégitimation de la RDA en montrant qu'elle n'est pas réductible à la Stasi. Pour reprendre le mot de Sandrine Kott et Martine Mespoulet, " Le communisme ne peut être analysé comme une sorte de prélude au post-communisme » (2006: 8). 


\section{Bibliographie}

Appenzeller, Gerd (2008), « DDR-Geschichte Lehren, was war », in Tagesspiegel, 29/07/2008.

Arp, Agnès / Leo, Annette (éd.) (2009), Mein Land verschwand so schnell...: 16 Lebensgeschichten und die Wende 1989/90, Weimar, Weimarer Taschenbuch Verlag.

Bessel, Richard / Jessen Ralph (dir.) (1996), Die Grenzen der Diktatur. Staat und Gesellschaft in der DDR, Göttingen, Vandenhoeck \& Ruprecht.

Dreyfus, Michel (dir.) (2000), Le siècle des communismes, Paris, Éditions de l'Atelier.

Droit, Emmanuel / Kott, Sandrine (dir.) (2006), Die ostdeutsche Gesellschaft. Eine transnationale Perspektive, Berlin, Christoph Links Verlag.

Engler, Wolfgang (2002), Die Ostdeutschen als Avantgarde, Berlin, Aufbau-Verlag.

Fabre-Renault, Catherine / Goudin, Élisa / Mesnard-Hähnel, Carola (éd.) (2006), La RDA au passé présent. Relectures critiques et réflexions pédagogiques, Paris, Presses de la Sorbonne nouvelle.

Faulenbach, Bernd (2003), " Nur eine ,Fußnote der Weltgeschichte'? Die DDR im Kontext der Geschichte des 20. Jahrhunderts ", in Eppelmann, Rainer / Faulenbach, Bernd / Mählert, Ulrich (éd.): Bilanz und Perspektiven der DDR-Forschung, Paderborn, Schöningh, 2003, 1-27.

Hein, Dörte (2004), « DDR-Geschichte im Internet. Zur Konstruktion eines multimedialen Gedächtnisses », in Hüttmann / Mählert Pasternack, op. cit., 265-290.

Hellmund, Saskia (2015), La fille qui venait d'un pays disparu, Paris, Les points sur les i.

Heß, Pamela (2014), Geschichte als Politikum. Öffentliche und private Kontroversen um die Deutung der DDR-Vergangenheit, Baden-Baden, Nomos Verlag.

Heukenkamp, Ursula (2004), « Warum und zu welchem Ende sollte man DDR-Literatur lehren? Probleme nachträglichen Verstehens ", in Hüttmann / Mählert / Pasternack, op. cit., 233-246.

Hübner Peter (éd.) (1995), Niederlausitzer Industriearbeiter 1935 bis 1970, Berlin, Akademie Verlag.

Hübner, Peter (1994), Konsens, Konflikt und Kompromis, Berlin, Akademie Verlag.

Hüttmann, Jens / Mählert, Ulrich / Pasternack, Peer (éd.) (2004) DDR-Geschichte vermitteln, Ansätze und Erfahrungen in Unterricht, Hochschullehre und politischer Bildung, Berlin, Metropol.

Hüttmann, Jens (2004), « Deutungskonflikte über DDR-Geschichte aus Akteursperspektive », in Hüttmann / Mählert / Pasternack, op. cit.,185-200.

Jarausch, Konrad H. (1995), " Die DDR denken. Narrative Strukturen und analytische Strategien ", in Berliner Debatte INITIAL 4/5, 9-15.

Köpping Petra (2018), Integriert doch erst einmal uns! Eine Streitschrift für den Osten, Berlin, Christoph Links Verlag.
Kott, Sandrine (2001), Le communisme au quotidien. Les entreprises d'Etat dans la société est-allemande, Paris, Belin.

Kott Sandrine (2000), "Collectifs et communauté dans les entreprises de RDA. Limites de la dictature ou dictature des limites? ", in Genèses, 2 (39), 27-51.

Kott Sandrine (1999), " La RDA, un Kulturstaat ouvrier ? », in Documents (1), 49-55

Kocka, Jürgen (1994), " Eine durchherrschte Gesellschaft », in Kaelble, Hartmut / Kocka, Jürgen / Zwahr, Hartmut (éd.), Sozialgeschichte der DDR, Stuttgart, Klett Cotta, 547-554.

Kott, Sandrine / Mespoulet, Martine (2006), Le post-communisme dans l'histoire, Bruxelles, Editions de l'Université de Bruxelles.

Lindenberger, Thomas (éd.) (1999), Herrschaft und Eigen-Sinn in der Diktatur. Studien zur Gesellschaftsgeschichte der DDR, Cologne, Böhlau.

Lüdtke, Alf / Kalinowski, Isabelle (1998), « La République démocratique allemande comme histoire. Réflexions historiographiques", in Annales. Histoire, Sciences Sociales (1) 53, 3-39.

Lüdtke, Alf (éd.) (1989), Alltagsgeschichte. Zur Rekonstruktion historischer Erfahrungen und Lebensweisen, Frankfort, Campus.

Meuschel, Sigrid (1993), Legitimation und Parteiherrschaft. Zum Paradox von Stabilität und Revolution in der DDR 1945-1989, Francfort, Suhrkamp.

Müller-Zetzsche, Marie (2018), Enseigner la RDA. La transmission de l'histoire de la RDA en Allemagne et en France après 1990, thèse soutenue à Leipzig sous la direction de Ulrich Pfeil et Thomas Höpel.

Pasternack, Peer (2004), « Wozu die DDR lehren? », in Hüttmann / Mählert / Pasternack, op. cit., 163-184.

Pasternack, Peer (2001), Gelehrte DDR. Die DDR als Gegenstand der Lehre an deutschen Universitäten 1990-2000, Wittenberg, Institut für Hochschulforschung.

Pilon, Nathalie (2012), « Enseigner la RDA : un défi de taille », in Documentation et bibliothèques, (3) 58, 154-159.

Rauhut, Michael (1993), Beat in der Grauzone. DDR-Rock 1964 bis 1972 - Politik und Alltag, Berlin, BasisDruck.

Sarovic, Alexander (2016), " Jugoslawienkrieg, Antworten auf die zehn wichtigsten Fragen », in Spiegel, 28/06/2016.

Schmiechen-Ackermann, Detlev (2002), Diktaturen im Vergleich. Kontroversen um die Geschichte, Stuttgart, Wissenschaftliche Buchgesellschaft.

Siemens, Ansgar (2015), « Kriminologe Christian Pfeiffer. Der Provokateur tritt ab », in Spiegel-Online, 25/03/2015.

Silbermann, Marc / Wrage, Henning (éd.) (2014), DEFA at the Crossroads of East German and International Film Culture, Boston, De Gruyter.

Staadt, Jochen (1996) " Eingaben: Die institutionalisierte Meckerkultur in der DDR, Goldbrokat, Kaffee-Mix, Büttenreden, Ausreiseanträge, und andere Schwierigkeiten mit den Untertanen », Arbeitspapiere des Forschungsverbundes SED-Staat, FU Berlin, $N^{\circ} 24$ 